\title{
An Intelligent MAC Protocol Selection Method based on Machine Learning in Wireless Sensor Networks
}

\author{
Mu Qiao, Haitao Zhao*, Shengchun Huang, Li Zhou and Shan Wang \\ College of Electronic Science, National University of Defense Technology \\ Changsha, China \\ [e-mail: \{qiaomu16, haitaozhao, huangsc, zhouli2035, chinafir\}@nudt.edu.cn] \\ *Corresponding author: Haitao Zhao
}

Received September 26, 2017; revised March 11, 2018; revised April 18, 2018; accepted May 21, 2018; published November 30, 2018

\begin{abstract}
Wireless sensor network has been widely used in Internet of Things (IoT) applications to support large and dense networks. As sensor nodes are usually tiny and provided with limited hardware resources, the existing multiple access methods, which involve high computational complexity to preserve the protocol performance, is not available under such a scenario. In this paper, we propose an intelligent Medium Access Control (MAC) protocol selection scheme based on machine learning in wireless sensor networks. We jointly consider the impact of inherent behavior and external environments to deal with the application limitation problem of the single type MAC protocol. This scheme can benefit from the combination of the competitive protocols and non-competitive protocols, and help the network nodes to select the MAC protocol that best suits the current network condition. Extensive simulation results validate our work, and it also proven that the accuracy of the proposed MAC protocol selection strategy is higher than the existing work.
\end{abstract}

Keywords: MAC protocol; machine learning; classification; competitive protocol; non-competitive protocol

This research was supported in part by the National Natural Science Foundation of China [Grant No. 61471376, No. 61601482, No. 61501482]. We express our thanks to Prof. Xianghui Cao who checked our manuscript. 


\section{Introduction}

Medium Access Control (MAC) protocols play an important role in wireless sensor networks. It also has been widely used in Internet of Things (IoT) applications to support large and dense networks [1]. In dense networks, sensor nodes always carry out single and simple MAC protocol. According to different application requirements, these MAC protocols can be generally classified into competitive protocols (e.g. CSMA/CA), non-competitive protocols (e.g. TDMA) and hybrid protocols. Common sensor nodes are usually tiny and inexpensive devices with a small battery. However, beacuse the existing multiple access methods for improving protocol performance prefers the optimal adaptation-based approach, they are not affordable for the senor nodes due to the heavy computational burden.

Existing researches and practices have shown that the competitive protocols are more suitable for the network with lighter loads, while the non-competitive protocols are more suitable for the network with heavier loads [2],[3]. Therefore, researchers have proposed several hybrid strategies to deal with the constraint of a single MAC protocol under the dynamic network environment [4],[5]. The fundamental problem of the above issues is that the principle of protocol selection is ambiguous to some extent. Considering different parameters, the decision may be contradictory under different scenarios, and the complicated algorithm usually caused varying degrees of control overhead. The development of machine learning make itself a potential solution for these problems.

Recently, the machine learning technique has been adopted to improve MAC protocols. The work in [6],[7] focuses on Primary User's (PU's) power and time feature, and uses the Support Vector Machine (SVM) to train the classifier to identify the current protocol of PU. The classification result can help the Secondary User (SU) to choose the right network according to its requirement. Naddafzadeh et al. [8] and Zhou et al. [9] investigate reinforcement learning schemes to help SUs capture the state of the primary user, and learn the reasonable feedback to improve its own utility. All the aforementioned work focuses on the PU's inherent behavior and only considers the power or the statistical features of traffic load. In other words, the existing studies ignore the dynamic network environments and the external characteristics of other items. Therefore, we exploit the intelligent technique to put forward a solution to the application limitation problem of the single type MAC protocol in wireless sensor networks.

In this paper, we propose a MAC protocol selection model for the dynamic network environment. Some crucial network parameters are chosen to build the feature data set, and a classifier is trained based on the classification learning technique. The MAC protocol selection model can select optimal MAC protocol when the classification accuracy becomes desirable. We present a selection framework, which completes the MAC protocol selection task through two stages. The first stage is classification learning process, where different kinds of feature data sets are trained by Sequential Minimal Optimization (SMO) algorithm to obtain an optimal classification result. The second stage is the selection decision process, where the classifier will help network nodes to select a suitable MAC protocol. Firstly, we collect the external environment parameters and the inherent parameters of the network to build a data set in the learning process. Meanwhile, for the two-class problem, we chose SMO algorithm with polynomial kernel to train the data set. After that, we obtain a classifier in the decision process. Finally, the sensor node can use the classifier to decide whether the current MAC protocol should be changed. The experimental results show that classification performance is significantly better than existing solutions. To the best of our knowledge, the proposed 
selection model is the first intelligent method that utilizes classification learning for MAC protocol selection. The main contributions of this paper can be summarized as follows:

- We present a MAC protocol selection model based on machine learning which aims at selecting the most suitable MAC protocol.

- We use the fuzzy set theory to improve the decision criterion, by this means a robust and effective protocol selection model is obtained.

- We use multi-granule incremental knowledge gaining technique to build a data set for training, which can gradually improve the accuracy of classification result.

The remainder of this paper is organized as follows. Section 2 briefly reviews related work. Section 3 introduces the system model. Section 4 explains the classification learning method for MAC protocol selection. Section 5 reports the validation of our proposal via extensive simulations. Finally, Section 6 concludes this research.

\section{Related Work}

A single type MAC protocol has limited application scope in the dynamic network environment, which is a key challenge in the design of wireless sensor network. This challenge has been attracted extensive investigation over the past decades, and the related proposals can be broadly divided into two categories. The first category [4],[5],[10]-[17] improves MAC protocol performance optimization through the traditional optimization algorithms, and the second category [6]-[9],[18],[19] accomplish this task by exploiting intelligence techniques.

For the first category, most of the access strategies modify the protocol frame structure adaptively to fit the dynamic network environment. The first approach to achieve this goal is to improve the performance of competitive protocols via dynamic adjustment of collision window and packet aggregation, or other collision handling schemes. Recently, such an idea has been upgraded by adopting learning ability [10]-[12]. Jang et al. [10] discuss a fully-distributed CSMA game-theoretic algorithm for each link without message exchange. Users can follow an ordinal potential game function to improve throughput performance. Stamatakis et al. [11] propose a two-stage dynamic spectrum assignment schemes to support more access opportunities for SU. Pandit et al. [12] present an event-driven backoff algorithm to minimize collision among the cooperation cognitive users when someone has been reserved the idle channels. However, these protocols are still not sufficient to support dense networks. The second approach is to improve the performance of non-competitive protocols by using more efficient MAC scheduling algorithms and policies to assign time slots to each real-time node to send their traffic. Liu et al. [13] propose a distributed multi-channel topology-transparent broadcast scheduling algorithm in Ad hoc networks. In [14], Sami et al. investigate a cooperative MAC protocol for CRNs with opportunistic energy harvesting in SUs. However, these protocols require high computational complexity and exact network topology. The third approach is based on the idea of hybrid access strategies that combine the advantage of the aforementioned protocols to solve competing and scheduling problems. Zhou et al. [15] dynamically adapt the duty-cycle according to the current network traffic, in which, the dynamic TDMA slots structure can be modified when the traffic increases significantly. According to [16], a novel hybrid MAC protocol can flexibly adapt to traffic and topology changing. Nodes can change their MAC behavior, because it allows contention in TDMA slots to cope with high traffic and low latency when some emergency events have been occurred. Ye et al. [17] propose an analytical model for DCF and dynamic-TDMA protocol to calculate MAC switching point in a tractable way. According to the switching point, nodes can change protocol boundary between DCF and dynamic-TDMA protocol under a time-varying network. 
Nevertheless, the main deficiencies of these studies are the excessive utilization of control information among nodes, which oppose the alleviation of communication overhead.

Among the second category, some intelligent protocols are proposed to optimize the protocol performance. Hu et al. [7] add the durations of PU's busy and idle times to the classification feature variables, so as to help the secondary user to choose the appropriate network according to its requirement. However, this research is mostly design an identification scheme for $\mathrm{SU}$, it is not directly optimize protocol performance. On the other hand, reinforcement learning has been used to improve the network throughput. Mihaylov et al. [18] propose a reinforcement learning approach, in which the synchronization reward signal can help nodes to transmit data and avoid inherent interferences. Followed by this work, [19] deepens a SVM based approach in a multi-channel and multi-hop network instead of the original single channel operation. Although the prior work has been learned from the perspective of the network environment and the node behavior to optimize protocol, most of them consider the learning target only from traffic load or energy while the valuable parameters in the presence of dynamic network environments have not been covered.

In this research, we use the classification learning method to deal with the application limitation problem introduced by the single type MAC protocol, where distinguishing the characteristics of different kinds of MAC protocols serves as one of the kernel tasks. This finding drives us to choose some typically used competitive protocol and the non-competitive protocol as candidates and jointly consider the impact of inherent behavior and external environments. On this basis, the classification learning process is the other important part.

\section{System Model}

There are two types of node in the considered system: access point (AP) and sensor node. We assume that there are only one AP and $N$ sensor nodes in the network. The AP is used to carry out classification learning task, which is equivalent to a supervisor in supervised learning. Since sensor nodes are tiny and inexpensive devices with limited hardware resources and power supply, they cannot support such learning computations. All of the nodes share a single wireless channel to transmit packets. In general, nodes are synchronized to the same timing clock, which can be achieved by global synchronization approaches, such as GPS, centralization synchronization [5], and mutual synchronization [2]. In our work, each node can adjust its own clock by receiving broadcast synchronization message from the AP. The broadcast information also includes the classification learning result. It can be used to help sensor nodes to select optimal MAC protocol when the classification accuracy achieves an acceptable result. We assume that: 1) the wireless channels are ideal, and 2) receiving errors solely stem from the message conflicts, and 3) each node always has a packet to transmit. When multiple messages arrive at a node simultaneously, all the messages are damaged, i.e., capturing effect is not considered.

\subsection{Framework for MAC Selection}

In the considered network, the nodes will notice AP when they access or leave the network. The notice messages include external parameters and network parameters information. Therefore, AP is used as a decision maker which can obtain the situation of the network. The flow chart of the MAC protocol selection framework is shown in Fig. 1, which is composed of two stages, i.e., learning process and decision process. 


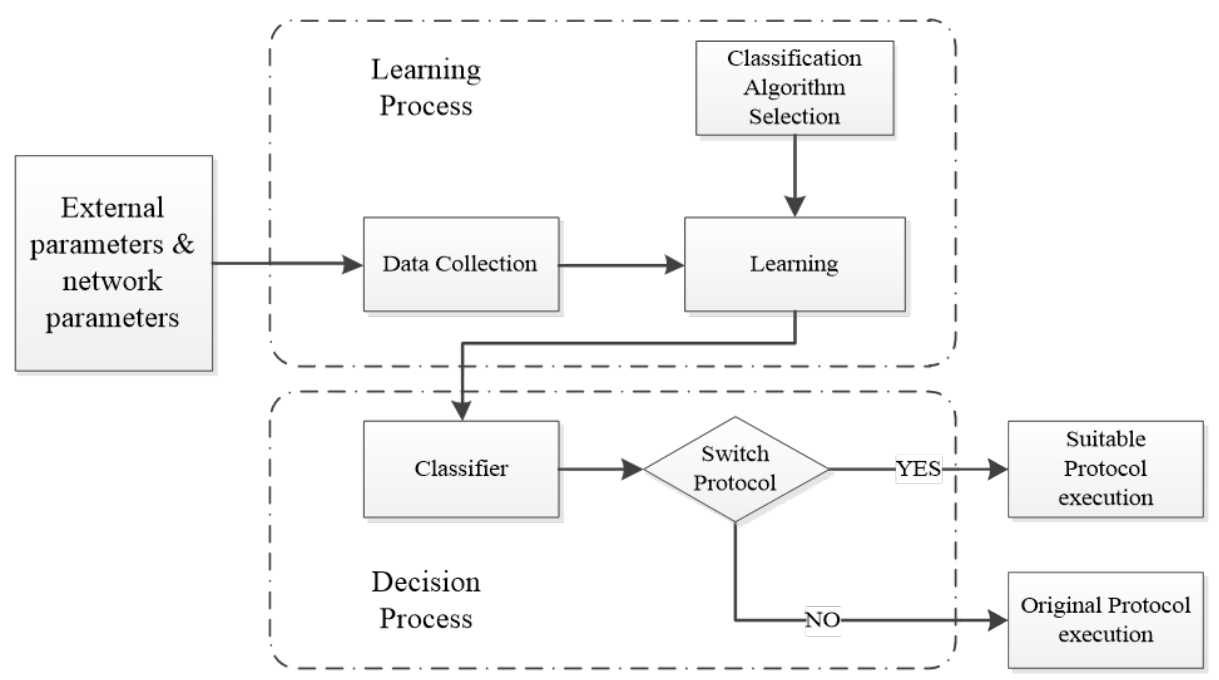

Fig. 1. The MAC protocol selection framework

\subsection{Differences between MAC Protocols}

In highly dynamic wireless network environments, the performance of competitive MAC protocol will be dramatically degraded when the network load becomes heavier. By contrast, the non-competitive protocol may waste network resources when the network load turns lighter. Taking account of this prior knowledge, we respectively select CSMA/CA and TDMA as the candidate MAC protocols to represent the competitive protocol and the non-competitive protocol in this paper. The performances of these two simple protocols vary significantly under different network conditions. For better understanding, we take a simple example as follows.

CSMA/CA protocol can be used in the distributed applications, as each node decides its time of accessing to the network independently. However, the competitive accessing strategy makes CSMA/CA protocol suitable for the networks with light load only [20]-[22]. Alternatively, TDMA protocol assigns the time slot to each sensor node in mandatory even if nodes do not send data. For the light traffic load, the majority of the time slots will be wasted and the throughput will remain at a low range. If the load is increased, all of the time slots will be saturated without collision [23]-[27]. So that, the throughput can rises to an efficient and stable state.

This paper investigates the performance differences of the two protocols under different network environments. By executing different MAC protocols, the input and output parameters of the network are collected to form a feature data set, which is further applied to establish the relationship between the network performance and the environment parameters. The relationship is then used to train a classifier to decide the suitable MAC protocol under different network loads. The evaluated classifier is finally used to help nodes to select the suitable protocol when the network environment varies with time.

\section{Classification Learning Method for Protocol Selection}

Taking account of the performance differences between competitive and non-competitive MAC protocol, we formulate the classification learning method in the protocol selection model. Based on this model, we establish a data set for training by using the multi-granule incremental knowledge gaining technique. Subsequently, the collected data set is applied to 
train the classifier.

\subsection{Protocol Selection Criterion}

As mentioned above, the CSMA/CA and dynamic TDMA are the candidate MAC protocols in this paper. In CSMA/CA, the node first detects whether the channel as idle. Then, it takes binary exponential backoff (BEB) algorithm opportunistic to access channel in collision avoidance phase. In dynamic TDMA, nodes are allocated with the time slot by reserving mutually and the nodes use the slots for packet transmission. The performance of the two protocols is compared in Fig. 2 with varying network loads. Obviously, CSMA/CA shows the better throughput performance with light load area, and TDMA shows the better throughput performance with heavy load area. As show in Fig. 2, the two performance curves cross at point $C$, the CSMA/CA curve reaches the maximum value at point $M$. Let point $C$ be the center, we introduce a symmetric point $M^{\prime}$ that satisfies $|M C|=\left|C M^{\prime}\right|$. Notably, on the left area of point $C$, the performance gap between CSMA/CA and TDMA is narrowed when the network load increases. While on the right area of point $C$, the performance gap between CSMA/CA and TDMA becomes widen with the increasing network load.

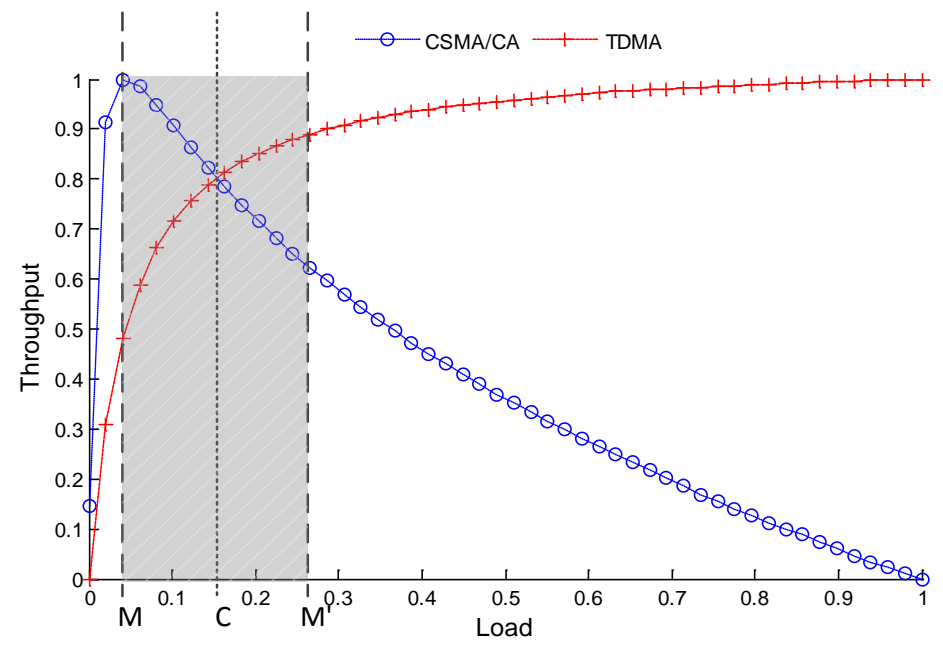

Fig. 2. The illustration of MAC protocol performances under different network loads

For the CSMA/CA curve, its performance decreases rapidly after passing through the point $M$, however, it is still better than TDMA counterpart before reading point $C$. Therefore, the classification criterion is decided by the crossing point rather than the maximum point. In order to gain the suitable protocol, it is desired to select and switch protocol is at point $C$. However, the protocol selection scheme may not always carry out timely. Consequently, we use the fuzzy set theory to circumvent this issue.

Definition 1: A fuzzy set is defined as $A=\left\{X_{i}\right\}, i \in N$, where $X_{i}$ stands for the pertaining result of node $i$. It can be expressed as:

$$
\begin{gathered}
X_{i}=\frac{\mu_{C S M A / C A}(x)}{x_{C S M A / C A}}+\frac{\mu_{T D M A}(x)}{x_{T D M A}}, \\
\text { s.t. } x_{C S M A / C A}, x_{T D M A} \in\{0,1\}, \mu \in[0,1]
\end{gathered},
$$


where $\mu_{\text {CSMA/CA }}$ and $\mu_{\mathrm{TDMA}}$ respectively represent the current protocol pertain to CSMA/CA and TDMA protocol. We use $\mu_{\text {CSMA/CA }}(x)$ and $\mu_{\text {TDMA }}(x)$ to denote the pertaining degree of current protocol, respectively. $x_{\text {CSMA/CA }}\left(x_{\text {TDMA }}\right)$ is the Boolean variable to convey whether node $i$ select CSMA/CA (TDMA) protocol. At point $C, \mu_{\text {CSMA /CA }}(x)=\mu_{\text {TDMA }}(x)=0.5$. If the network load is in the heavy area, the pertaining result prefers TDMA protocol, in this case $\mu_{\text {CSMA/CA }}(x)<\mu_{\text {TDMA }}(x), \quad x_{\text {CSMA/CA }}=0$ and $x_{\text {TDMA }}=1 . \mu_{\text {CSMA/CA }}(x)$ gradually decreases to 0 , $\mu_{\text {TDMA }}(x)$ gradually increases to 1 . If the network load is in the light area, the pertaining result prefers CSMA/CA protocol, accordingly $\mu_{\text {CSMA /CA }}(x)>\mu_{\text {TDMA }}(x), x_{\text {CSMA/CA }}=1$ and $x_{\text {TDMA }}=0$. $\mu_{C S M A / C A}(x)$ gradually increases to $1, \mu_{\text {TDMA }}(x)$ gradually decreases to 0 . Obviously, the optimal protocol switching opportunity is at cross point $C$. However, before the pertaining degree decrease to 0 or increase to 1 , the fuzzy area is also can be accepted.

Theorem 1: The sum of the pertaining degrees always holds

$$
0<\left(\mu_{\text {CSMA } / C A}(x)+\mu_{\text {TDMA }}(x)\right) \leq 1 .
$$

Proof: The performance gaps between CSMA/CA and TDMA varies with the load. Without regard to the cross point, the current suitable protocol can be defined as

$$
\left\{\begin{array}{c}
\mu=\mu_{C S M A / C A}(x)+\mu_{T D M A}(x) \\
\arg \max \left(\mu_{C S M A / C A}(x), \mu_{T D M A}(x)\right)>0.5 \\
\mu-\arg \max \left(\mu_{C S M A / C A}(x), \mu_{T D M A}(x)\right)<0.5 \\
\text { s.t. } x \in\{0,1\}, \mu \in[0,1]
\end{array} .\right.
$$

According to the pertaining degree of candidate MAC protocols, one is more than 0.5 and the other one is less than 0.5 .

Therefore, the sum of pertaining degree must be satisfied (2). Theorem 1 is proved.

\subsection{Collection of the Data Set}

When formulating the protocol selection model, the key issue is to unfold the relationship between the decision-making criterion and the network features (i.e. external and internal). Therefore, data collection is essential for the learning process. In order to validate the proposed scheme, we use two kinds of data sets, i.e., the feature data set for training, and the test data set for evaluation (the detailed experimental settings are presented in Section 5). The two kinds of data sets have the same feature items, while they include different number of samples. It is also worth noting that the features item includes both parametric feature and statistics features.

We selected 13 feature items to build data set. 6 of the 13 feature items in a data set are parametric features for protocol settings (i.e., protocol type, packet length, data rate, transmission inter-arrival time, transmit power, and node number). In addition, another 6 feature items are statistic features for performance evaluation (i.e., average load, delay, average throughput, min throughput, max throughput, and standard deviation throughput). Moreover, the feature item 'result' corresponds to the classification result. The detailed interpretations are given as below:

The 6 parametric features are general parameters for protocol settings, and they are widely adopted in many network simulation platforms, such as OPNET.

1). protocol type: It usually represents a current behavioral MAC protocol of a node.

2). packet length: It is not the size of the packet stored in the node's memory, but the modeled size for calculating transmission time, error probability, etc..

3). data rate: It specifies the information rate over the data transmission channel. 
4). transmission inter-arrival time: It represents the time interval between the two successive transmissions.

5). transmit power: It specifies the power consumed by packet transmission.

6). node number: It specifies the total number of nodes throughout the network.

The 6 statistic features involves 3 kernel performance metrics (i.e., load, delay, and throughput). Among them, throughput is a most important performance metrics. Therefore, we select different calculation method to consider this statistic feature (i.e. average throughput, min and max throughput, and standard deviation throughput).

1). delay: It represents the transmission delay of an individual packet from the source to the destination.

2). average load: It represents the total traffic streams which have been transmitted.

3). average throughput: It represents the average number of bits transmitted per second.

$4 \& 5$ ). min and max throughput: They describes the dynamic range of throughput.

6). standard deviation throughput: The variance is to measure how far, on average, a given throughput is from the average throughput. The standard deviation is the square root of the variance.

Table 1. Parametric Features

\begin{tabular}{|c|l|}
\hline Feature & \multicolumn{1}{c|}{ Description } \\
\hline \hline Protocol Type & $\begin{array}{l}\text { The protocol type which nodes are used in the current } \\
\text { networks }\end{array}$ \\
\hline Packet Length & Setting packet length in each simulation samples \\
\hline Data Rate & Setting data rate in each simulation samples \\
\hline $\begin{array}{c}\text { Inter-arrival } \\
\text { Time }\end{array}$ & Setting the time interval of packet arrival in each sample \\
\hline Transmit Power & Setting transmit power in each sample \\
\hline Node Number & Number of nodes in the current networks \\
\hline Result & Classification result \\
\hline
\end{tabular}

In this paper, as can be founded in Table 1, six condition parameters and the classification result are selected to form the parametric feature data set. The classification result is a Boolean variable, i.e., 0 or 1 , to convey whether the MAC protocol should be modified to adapt to the current network.

Table 2. Statistics Features

\begin{tabular}{|c|l|}
\hline Feature & \multicolumn{1}{c|}{ Description } \\
\hline \hline Average Load & Sum of the average load in each independent sample \\
\hline $\begin{array}{c}\text { Average } \\
\text { Throughput }\end{array}$ & Sum of the average throughput in each independent sample \\
\hline Delay & Sum of the transmitting delay in each independent sample \\
\hline Min Throughput & Sum of the minimum throughput in each independent sample \\
\hline Max Throughput & Sum of the maximum throughput in each independent sample \\
\hline Stddev & $\begin{array}{l}\text { Sum of the throughput standard deviation in each independent } \\
\text { sample }\end{array}$ \\
\hline Result & Classification result \\
\hline
\end{tabular}

After setting the parameters according to Table 1, we can obtain a series of statistical results. Six statistic parameters and the classification result are collected to form the statistic feature data set, which is summarized in Table 2. 
Then, the two feature data sets are combined with multi-granule incremental knowledge gaining technique [28],[29], so as to obtain a robust MAC protocol classifier for learning.

Definition 2: A MAC protocol selection system is defined as $S=\langle U, R, V, f>$, where $U$ stands for the sample feature data set, $R=C \cup D$ is the union set of $C$ and $D$, where the set $C$ covers the feature attribute and the set $D$ specifies the classification result attribute, respectively, $D=\{0,1\} . V_{i}=\cup_{r \in R i} V_{r}$ is the set of attribute values that are related to sensor $i$, $V_{r}$ indicates the value scope of the feature attribute $r \in R_{i} . f_{i}: U \times R_{i} \rightarrow V_{i}$ is the correlation function.

Definition 3: In MAC protocol selection system $S=<U, R, V, f>,(a, v)$ represents the feature item in the system, where $a \in R, v \in V$, named Feature Granular (FG). $m(a, v)$ denotes the collection of the samples value in $U$, where $m$ stands for the meaning function. $((a, v), m(a, v))$ is defined as a complete sample in the system $S$, named FG node.

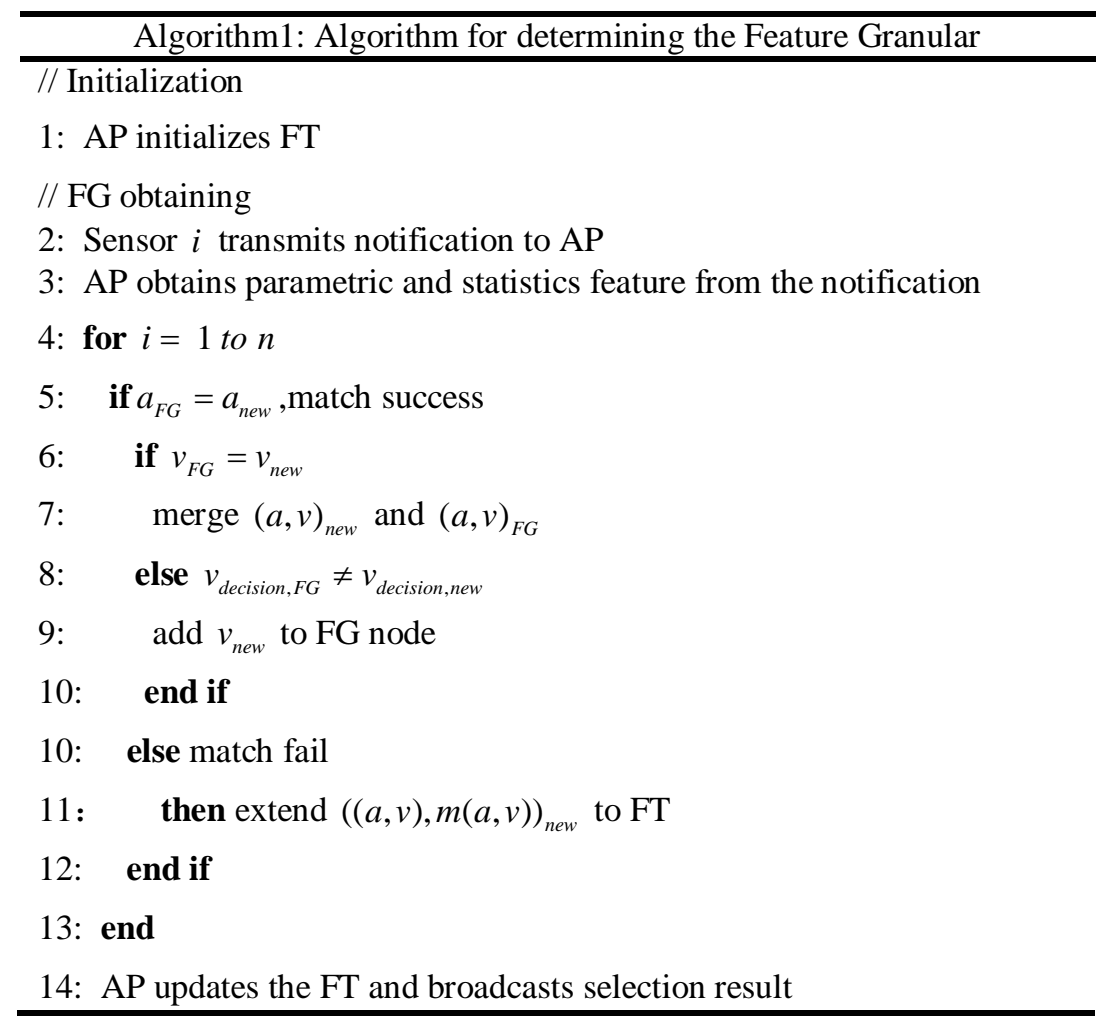

In order to speed up the searching process within the feature data set, we form a feature tree (FT) to quickly match the new samples. The FT is a tree-structured collection of FG samples according to the feature item. By this means, the FG can be searched along the tree layer-by-layer to find the leaf node corresponds to a certain sample value, named FG sub-node. In the FT, each layer is determined by a certain feature attribute $a(a \in R)$, each non-leaf node contains a feature item $(a, v)$ in which the attribute value $v$ only affects the sample size of attribute $a$. If a new arrival sample $s_{\text {new }}=((a, v), m(a, v))_{\text {new }}$ matches with an existing FG feature item accurately, we merge the new sample with the granule and update the FT. In addition, if feature item value of $s_{\text {new }}$ is different from existing FG node that keep the same 
feature item. The different attribute value should be added as a new sample. Otherwise, we extend a new sample to the tree as a new FG node.

In the initialization phase, AP initializes its FT. In the FG obtaining phase, sensor $i$ first transmits notification to AP, from which AP obtains parametric and statistics features. Next, AP needs to determine whether $s_{\text {new }}$ matches with existing FG node. In specific, $s_{\text {new }}$ can successfully be matched with FG node if and only if they have the same condition and decision attribute values. Under such a condition, we merge them together. Otherwise, we should deal with the object as a conflicted FG node. Furthermore, if $s_{\text {new }}$ fails to match with the FG node, we should create it to be an individual FG node. This training process is repeated until the classification accuracy achieves an acceptable result. Finally, AP updates the FT and broadcast the MAC protocol selection result. The above discussion is summarized in Algorithm 1, where the multi-granule incremental knowledge gaining technique has been adopted to obtain FG.

The computational complexity of Algorithm 1 is $O\left(\sum_{i=1}^{m} C_{m r}^{i}\left(\left|U^{\prime}\right|+1\right)^{i} \log _{2}\left(\left|U^{\prime}\right|+1\right)\right)$, where $\left|U^{\prime}\right|$ is the number of successful matching, $m$ is the number of conflicted FG node reduction, and $r$ is the number of maximum values in condition attribute set.

\subsection{Selection of the Classifier}

The SMO algorithm is an upgrade of the SVM method, and this rapid quadratic programming optimization algorithm is a linear SVM suitable for sparse data. Similar to SVM, the classification function of SMO can be expressed as

$$
f(x)=w^{T} x+b,
$$

where $f(x)=0$ represents the hyper plane. $w$ is weighing vector. $b$ is a constant value. The two-class problem of the two support vector can be denoted by

$$
\left\{\begin{array}{l}
w^{T} x+b=1 \\
w^{T} x+b=-1
\end{array}\right. \text {. }
$$

If the symbols of $w^{T} x+b$ and class symbols of $y$ are consistent, classification result can be considered as correct. To enable represent the distance, we introduce the definitions of functional margin and geometrical margin. Function margin is represented by

$$
\hat{\gamma}=y\left(w^{T} x+b\right)=y f(x) \text {. }
$$

Geometrical margin is expressed as

$$
\tilde{\gamma}=y \gamma=\frac{\hat{\gamma}}{\|w\|}
$$

where $\tilde{\gamma}$ is the geometrical margin which is the distance between a sample to the hyper plane. $\hat{\gamma}$ is the function margin which is the minimum value of all samples $\left(x_{i}, y_{i}\right)$ in the hyper plane $(w, b) . y$ is the class symbol (i.e. +1 or -1$)$.

For this classification problem, we need to maximize the margin to obtain a maximum margin classifier. The objective function can be

$$
\max \frac{1}{\|w\|} \text {, s.t. } y_{i}\left(w^{T} x_{i}+b\right) \geq 1, i=1, \cdots, n \text {. }
$$


which results in the following optimization problem:

$$
\begin{gathered}
\min _{\alpha} \Psi(\vec{\alpha})=\min _{\alpha} \frac{1}{2} \sum_{i=1}^{n} \sum_{j=1}^{n} y_{i} y_{j} K\left(x_{i}, x_{j}\right) \alpha_{i} \alpha_{j}-\sum_{i=1}^{n} \alpha_{i} \\
\text { s.t. } 0 \leq \alpha_{i} \leq C, i=1, \ldots, n \\
\sum_{i=1}^{n} \alpha_{i} y_{i}=0
\end{gathered}
$$

In this process, the SMO algorithm selects two factors $a_{i}$ and $a_{j}$ for regulating in each iteration, while other factors remain unchanged. Compared to the conventional decomposition algorithms, SMO algorithm may involve a larger number of iterations. However, the computation in each iteration is relatively simple. In addition, the SMO algorithm does not need to store the kernel matrix, thus there are no matrix operations.

It is worth mentioning that the linear inseparable problem should be solved by utilizing the kernel function. In order to solve the classification problem in the current space, we need to map the data to a high-dimensional space. The commonly used kernel functions are as follows:

$$
\left\{\begin{array}{l}
\text { PolynomialKernel: } K\left(x_{1}, x_{2}\right)=\left(<x_{1}, x_{2}>+R\right)^{d} \\
\text { Linear Kernel: } K\left(x_{1}, x_{2}\right)=<x_{1}, x_{2}> \\
\text { Gaussian Kernel: } K\left(x_{1}, x_{2}\right)=\exp \left(-\left\|x_{1}-x_{2}\right\|^{2} / 2 \sigma^{2}\right)
\end{array} .\right.
$$

With the varying proportion of data samples for training, the classification accuracy under different kernel functions is compared in Fig. 3. We select the ZeroR algorithm as the baseline. It can be found the performance achieved by the three SVM kernels is significantly better than the baseline. Moreover, the polynomial kernel outperforms the remaining two kernel functions. Thus we choose the polynomial kernel in the proposed classifier.

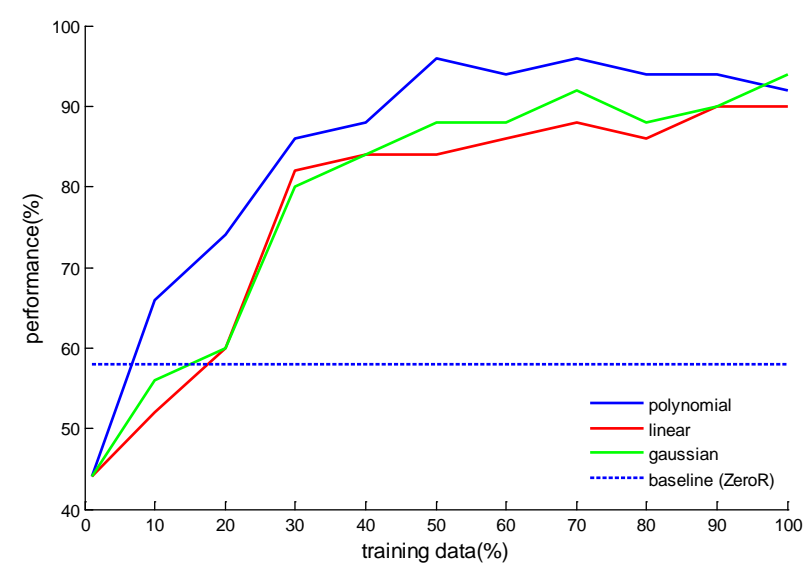

Fig. 3. Learning curve of different kernel function

\section{Evaluation}

\subsection{Simulation Setup}

By combining parametric and the statistical feature data sets, a data set containing 13 features can be obtained and used for classifier learning. Each sample contains both parametric and 
statistical features, and we vary the value of the parametric features, such as data package length (e.g. 100 1024 bytes), message transmitting rate (e.g. 1, 2, 5.5, $11 \mathrm{Mbps}$ ), interval time (e.g. 0.00001 0.01 seconds), transmit power (e.g. 0.001 1 W) and the node number (e.g. 5 30, It is incremented by 5 until it reaches 30 ). Some physical parameters are fixed, e.g., the direct sequence spectrum spreading technique is used in the physical layer, where the input Short Interframe Space (SIFS) and the DCF Interframe Space (DIFS) are respectively $10 \mu \mathrm{s}$ and $50 \mu \mathrm{s}$, the duration of a time slot is $20 \mu \mathrm{s}, c w_{\min }=31, c w_{\max }=1023$.

The classification result is obtained by comparing the samples with the saturation curve under the current network environment. If the pertaining degree of current MAC protocol holds $\mu(x)<0.5$, the classification result should be 0 and the protocol should be switched. Then, the feature data set is trained for classification, and to obtain the protocol selection model. We use the cross validation method with which to train the data set. The SMO algorithm with polynomial kernel is used to train the feature data set via 10-flod cross validation, and the weighted average PoCC is computed to evaluate the proposed MAC protocol selection model. According to the classification result, the network performance should be kept whether the network environment has been changed.

In order to validate the MAC protocol selection model, the experiment is divided into two phases. In the first phase, we collected samples in the feature data set and built the MAC protocol classifier. 1000 simulation samples are collected from OPNET simulation to train the classifier, where half of the samples are collected under CSMA/CA protocol while the rest half are under TDMA protocol. The size of data set is explained by the following two considerations: 1 ) the classification result of classifier is acceptable; 2 ) there are finite samples trained in AP node. In the second phase, we collect individual samples in the test feature data set and evaluate the performance of the classifier. The above experiment settings are followed here and one hundred simulation samples are collected as a test data set to evaluate the classifier. The samples associated with CSMA/CA and TDMA equally split the set. The training data set is generated from OPNET simulation. After setting the parametric feature items in Table 1, statistic results are collected via simulations. We establish the range of the parametric features, while in each simulation, the concrete settings are randomly selected within the range.

\subsection{Evaluation of Classifier and Feature Contribution}

Simulation results indicate that the weighted average probability of correct classification (PoCC) obtained is 94\%. In comparison, the corresponding baseline PoCC is 58\%. The proposed protocol selection model surpasses the baseline in the given dynamic network environment.

To enable evaluate the classifier, we test four conventional classification algorithms, i.e., NaiveBayes [31], J48 [30], SMO [32] and RandomForest [33]. We perform 10-flod cross validation on the feature data set. Each algorithm runs 10 times, and the PoCC shows in Fig. 4. (a). The results indicate that, PoCC of J48, NaiveBayes, SMO and RandomForest are $90 \%$, $94 \%, 94 \%$ and $96 \%$, respectively. 


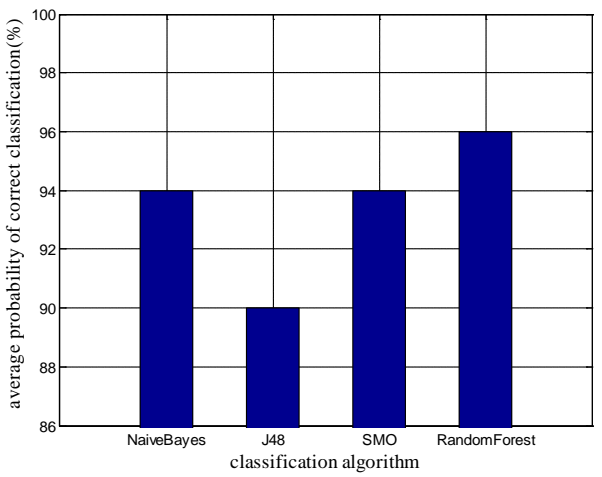

(a) Correct classification probabilities

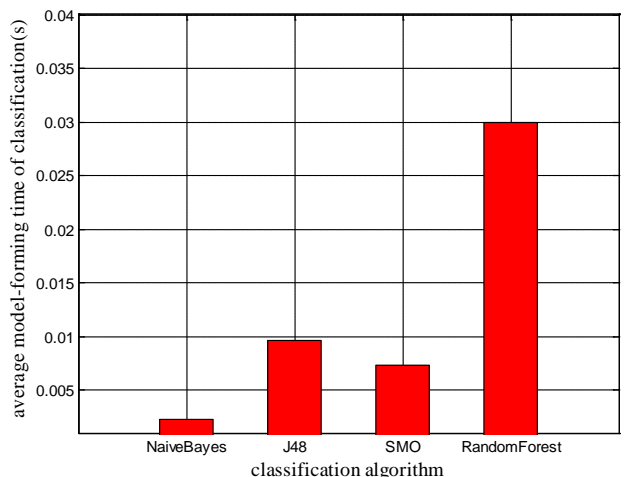

(b) Time for model training

Fig. 4. Comparison between different classifiers

The time for model training is shown in Fig. 4. (b). RandomForest algorithm achieves the highest PoCC among the four algorithms, however, it takes 30ms to train the protocol selection model with 100 decision trees, which is much longer than the other schemes and make the algorithm less efficient. The J48 algorithm obtains an 11-node decision tree after pruning, where 6 of them are leaf nodes. This result indicates that J48 may be suffer from over-fitting and is not suitable for extending to other network environments. Moreover, the features within the data set selection in this paper are correlated to some degree. When removing the last four lowest information gain ranking features as shown in Table 3 . The NaiveBayes algorithm becomes unstable. Therefore, we choose SMO algorithm to establish MAC protocol selection model in this paper.

Table 3. Comparison between the simplification classifiers

\begin{tabular}{|c|c|c|}
\hline Algorithm & Accuracy & Time \\
\hline \hline NaiveBayes & $94 \%(86 \% \downarrow)$ & $2.2 \mathrm{~ms}(1 \mathrm{~ms} \downarrow)$ \\
\hline J48 & $90 \%(90 \%-)$ & $9.6 \mathrm{~ms}(1.7 \mathrm{~ms} \downarrow)$ \\
\hline SMO & $94 \%(92 \% \downarrow)$ & $7.3 \mathrm{~ms}(4.6 \mathrm{~ms} \downarrow)$ \\
\hline RandomForest & $96 \%(94 \% \downarrow)$ & $30 \mathrm{~ms}(20 \mathrm{~ms} \downarrow)$ \\
\hline
\end{tabular}

There are two main factors that affect the performance of the MAC protocol selection model, one is feature data set collection, and the other one is the classification algorithm that can match with the characteristics of the data set. In this paper, 13 feature items are selected based on the analysis of the MAC protocol and the network formulation. Among the features, the feature item 'Result' corresponds to the classification result, and the other 12 ones are treated as conditional features. The PoCC is somewhat acceptable, while it should be further noted that, these features contribute differently to the performance of the MAC protocol selection. Therefore, it makes sense to select a subset of the features that are essential for the MAC protocol. In order to fully cognize the feature information, we should identify the contributions of different features to the final PoCC. We use (11) to calculate the information gain of the 12 conditional features in the feature set. The results are listed in Table 4. 


$$
\left\{\begin{array}{l}
\operatorname{Info}(D)=-\sum_{i=1}^{m} p_{i} \log _{2}\left(p_{i}\right) \\
\operatorname{Info}_{A}(D)=-\sum_{j=1}^{n} P\left(A_{j}\right) \sum_{i=1}^{m} P\left(C_{i} \mid A_{j}\right) \log _{2} P\left(C_{i} \mid A_{j}\right) . \\
\operatorname{InfoGain}(A)=\operatorname{Info}(D)-\operatorname{Info}_{A}(D)
\end{array}\right.
$$

Table 4. Information Gain Ranking of different features

\begin{tabular}{|c|c|c|}
\hline Rank & Feature & Information gain \\
\hline \hline 1 & Stddev Throughput & 0.596 \\
\hline 2 & Packet Length & 0.579 \\
\hline 3 & Average Throughput & 0.513 \\
\hline 4 & Max Throughput & 0.411 \\
\hline 5 & Delay & 0.305 \\
\hline 6 & Average Load & 0.232 \\
\hline 7 & Inter-arrival Time & 0.096 \\
\hline 8 & Data Rate & 0.037 \\
\hline 9 & Node Number & 0.011 \\
\hline 10 & Transmit Power & 0.007 \\
\hline 11 & Min Throughput & 0.00026 \\
\hline 12 & Type & 0.00008 \\
\hline
\end{tabular}

Form Table 4, the throughput, data package length and delay possess the highest information gain, which are directly correlated with the dynamic network. The ranking of different features indicates the requirement of different networks on the MAC protocol. Throughput refers to the amount of successfully-transmitted data. Maximizing the global throughput is a key object of the multiple access schemes. Delay refers to the time requirement for a packet to be transmitted successfully. Minimizing delay is a key performance metric for real-time traffic load.

On the other hand, the two highly-ranked parametric features are "Packet Length" and "Inter-arrival Time". The Table 4 also indicates that the information gains of the 8th to 12th features are much smaller than the others. As shown in Fig. 5, the information gain gradually decreases to 0 . Thus, the feature set can be simplified by removing features with low information gain. It can be used to save the time resources when delay is an important figure of merit for the network.

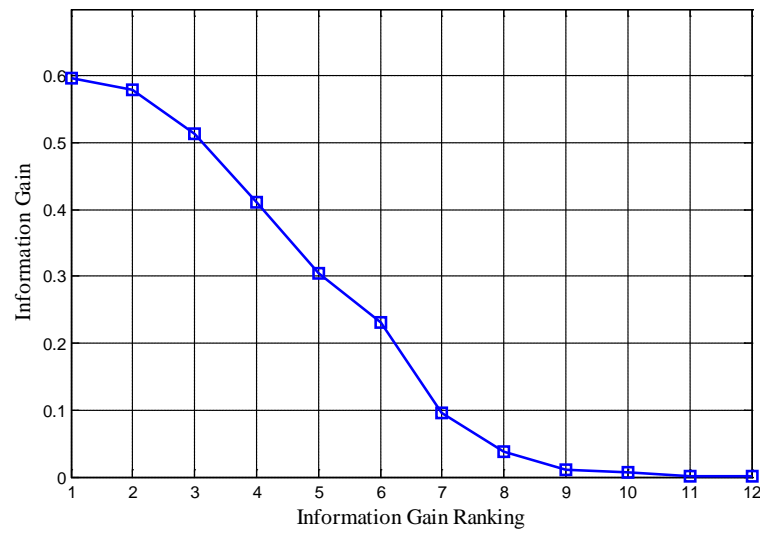

Fig. 5. Information gain of different features 


\subsection{Evaluation of Throughput Feature Distribution}

Fig. 6 shows the distribution of the protocol type in the node number-throughput plane. Moreover, Fig. 7 shows the distribution of the classification result in the plane, where blue circles indicate that the current MAC protocol should be preserved, while red crosses indicate that the protocol need to switch. By comparing the results in Fig. 6 and Fig. 7, it can be found that both the CSMA/CA and TDMA protocols have some samples which require to switch current MAC protocol. Moreover, Fig. 7 shows that the more red crosses appear when the number of nodes is increased for the CSMA/CA protocol, the more blue circles are appeared for the TDMA protocol. The reasons can be as follows: 1) the traffic load becomes heavier when the number of node increases, and 2) more nodes will encounter more severe conflicts.

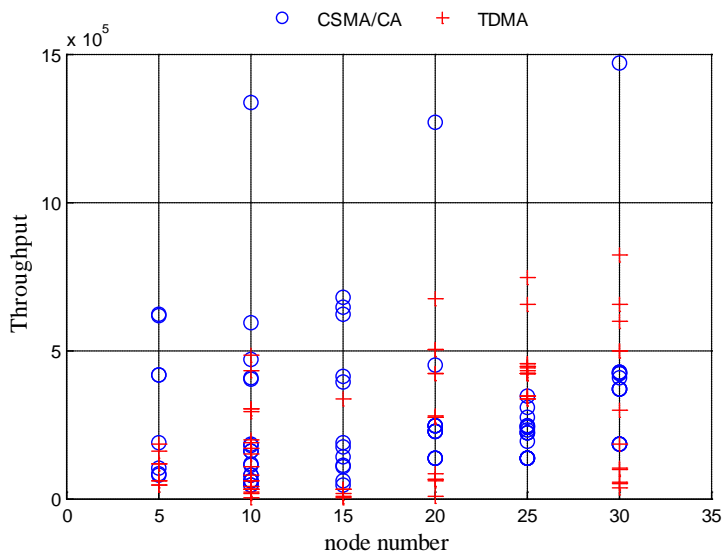

Fig. 6. Protocol type featured distribution

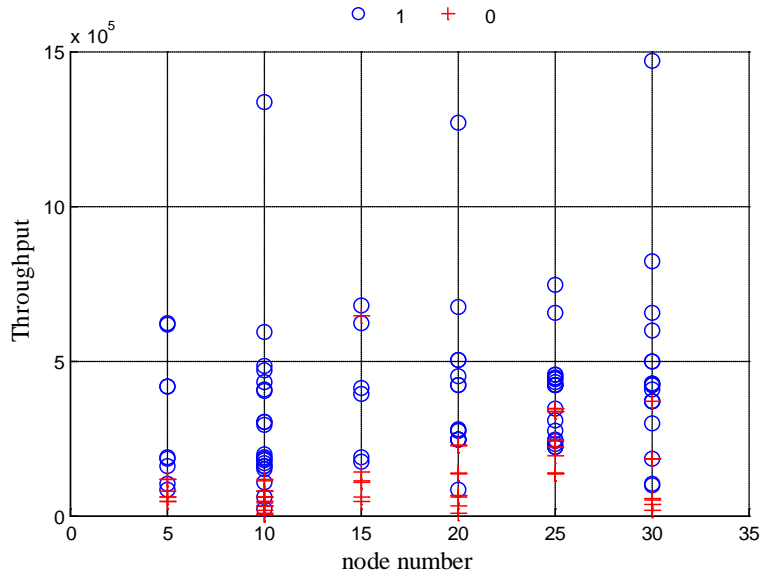

Fig. 7. Result feature distribution 


\subsection{Evaluation of Traffic Load Feature Distribution}

This part verifies the classification performance of the proposed model. To use the high rank contribution of statistics features in Table 5 (i.e. "Throughput", "Delay", and "Average Load"), we compare the candidate MAC protocol network performance and feature distributions. We use the individual test data set to evaluate the classifier and observe the performance of our proposed protocol selection model. We set the number of the network node to 5, 10, 15, 20, 25 and 30, respectively, while the size of the data packet at 1000 bytes. According to the effects of different feature in Table 4, we select standard deviation throughput, delay, and average load to evaluate the classifier in Figs. 8-13 under different network sizes. More explicitly, Figs. 8-13 (a) and (b) represent the performance difference between CSMA/CA and TDMA, and Figs. 8-13 (c) and (d) show the distribution of the classification results of average load feature.

First, in Fig. 8. (a) - Fig. 13. (a), and Fig. 8. (b) - Fig. 13. (b), we can observe that the performance of CSMA/CA is better than that of TDMA under the scenarios with 5, 10, and 15 nodes, while it degrades dramatically when the number of nodes exceeds 20 . We can roughly point out that CSMA/CA is more suitable when the network includes less than 20 nodes. Otherwise TDMA is a more suitable choice. The reason is that the competition becomes severer if adding more nodes to the network, which degrades the performance of CSMA/CA protocol. On the contrary, as TDMA is more suitable for heavy load scenarios, the relevant performance can be improved gradually when more nodes are operated in the network.

Second, we can observer the classification result distributions in Fig. 8. (c) - Fig. 13. c), and Fig. 8. (d) - Fig. 13. (d). Fig. 8. (c) - Fig. 13. (c), and Fig. 8. (d) - Fig. 13. (d) show the distribution of the classification results of average load feature, where Fig. 8. (c) - Fig. 13. (c) represent the sample distributions of nodes which use CSMA/CA protocol, Fig. 8. (d) - Fig. 13. (d) represent the sample distributions of nodes which use TDMA protocol. The blue circles and the red crosses comply with the definition in Fig. 7. In Fig. 8. (c) - Fig. 13. (c), when CSMCA/CA is adopted, the samples with "suitable" classification results are generally associated with light load. In Fig. 8. (d) - Fig. 13. (d), when TDMA is adopted, the samples with "suitable" classification result are generally associated with heavy load. By utilizing more nodes in simulations, the above distribution can be more evident. This is because TDMA will cause some redundant slots when the network stays in the light load scenario. However, CSMA/CA becomes not feasible when severer collisions are encountered in the heavy load scenario. Therefore, the protocol selection model can select the appropriate MAC protocol under different network loads. 


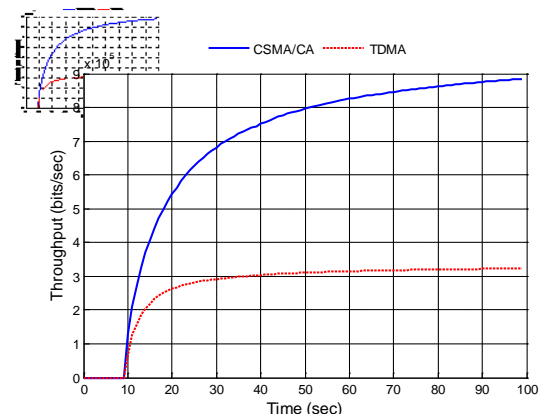

(a)

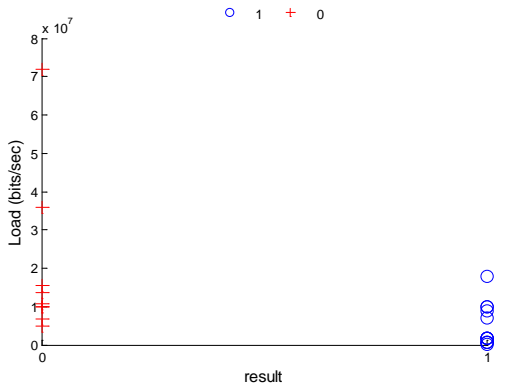

(c)

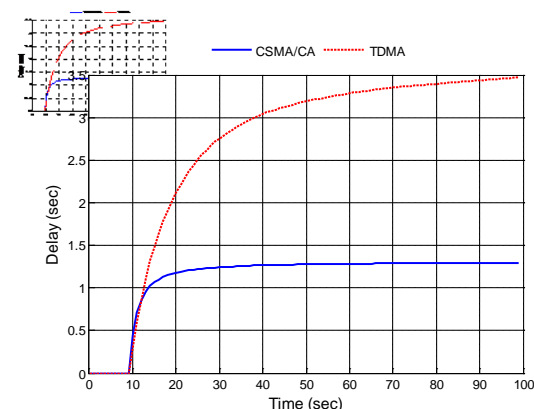

(b)

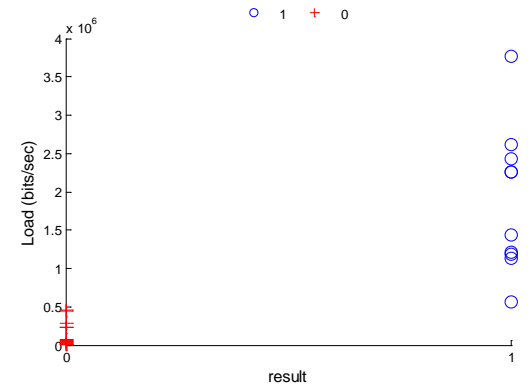

(d)

Fig. 8. Performance comparison between CSMA/CA and TDMA under 5 node scenarios. (a) throughput standard deviation. (b) delay. (c) CSMA/CA samples. (d) TDMA samples
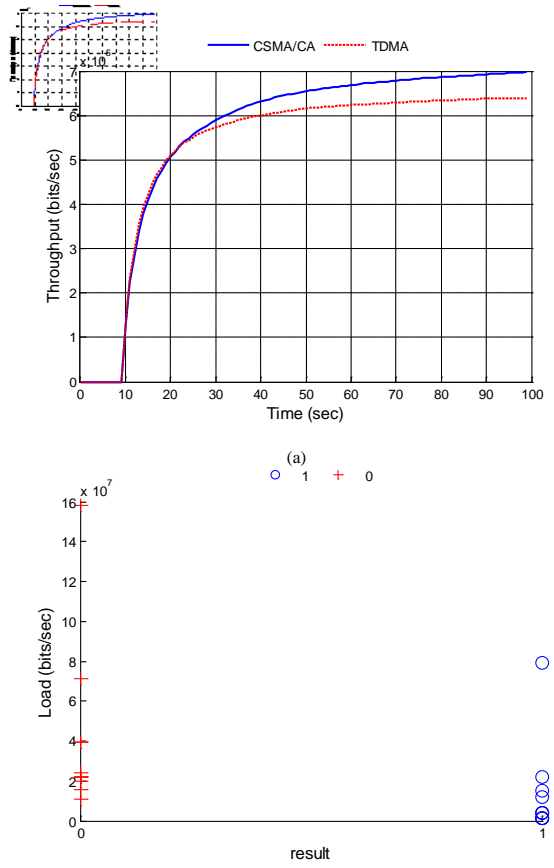

(c)
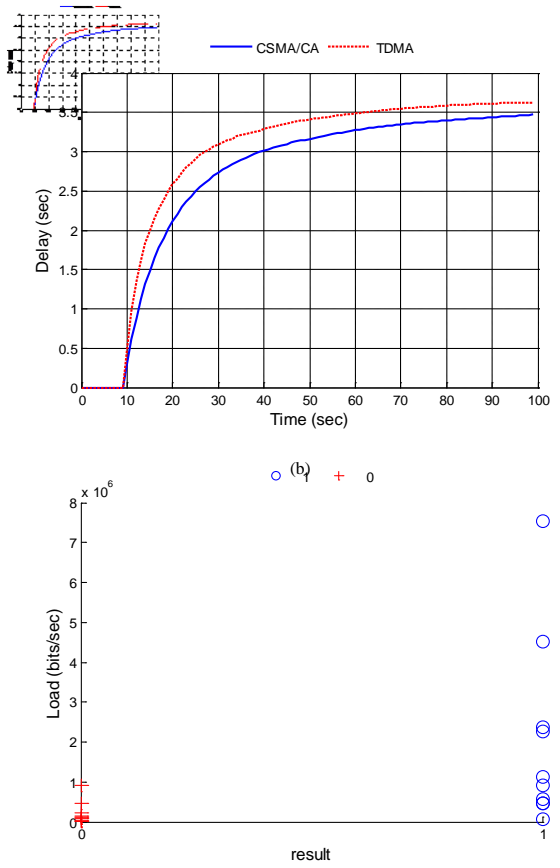

(d)

Fig. 9. Performance comparison between CSMA/CA and TDMA under 10 node scenarios. (a) throughput standard deviation. (b) delay. (c) CSMA/CA samples. (d) TDMA samples 


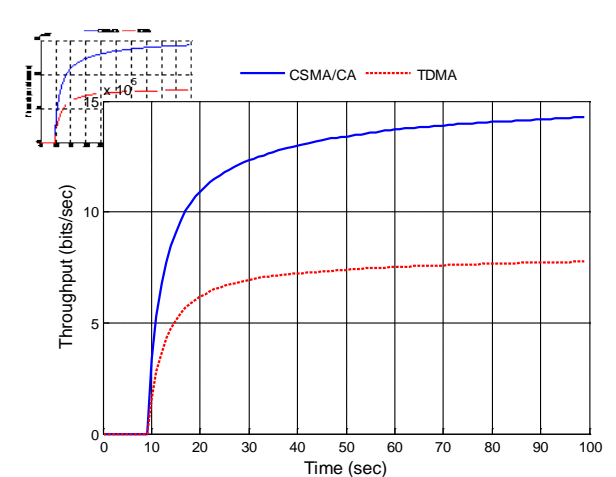

(a)

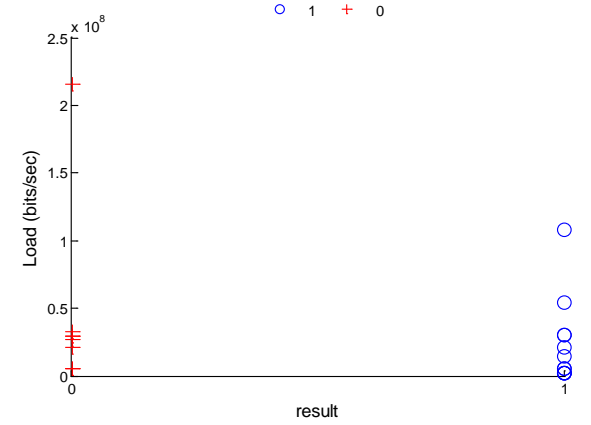

(c)

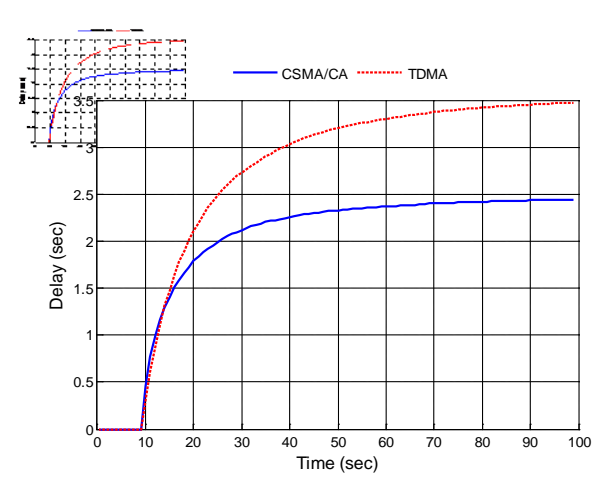

(b)

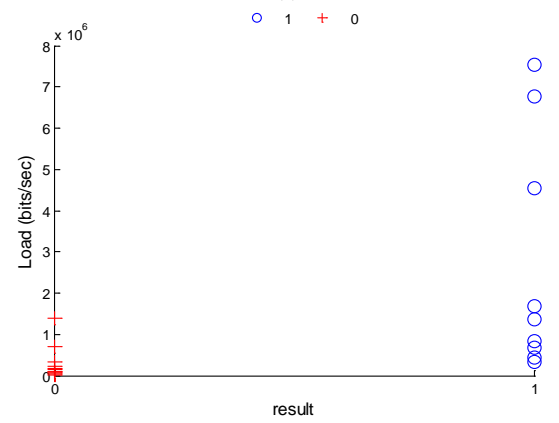

(d)

Fig. 10. Performance comparison between CSMA/CA and TDMA under 15 node scenarios. (a) throughput standard deviation. (b) delay. (c) CSMA/CA samples. (d) TDMA samples

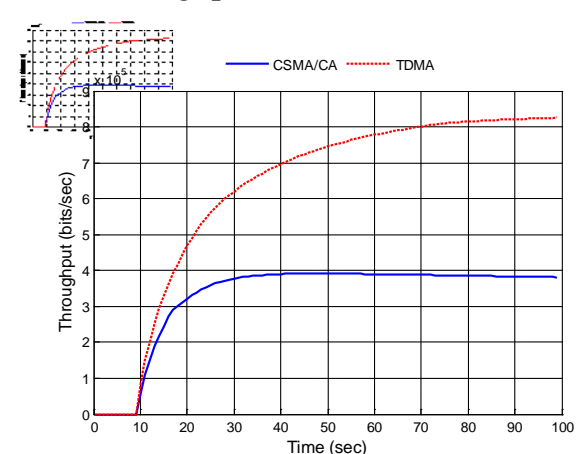

(a)

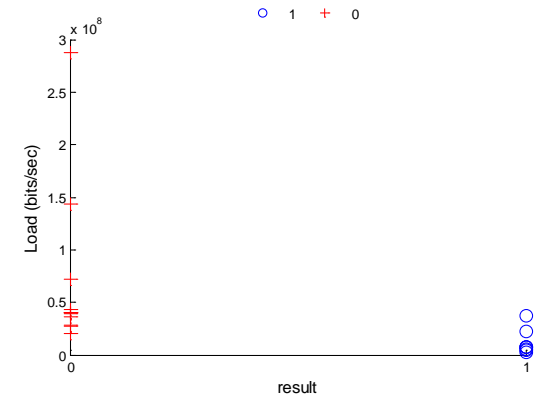

(c)

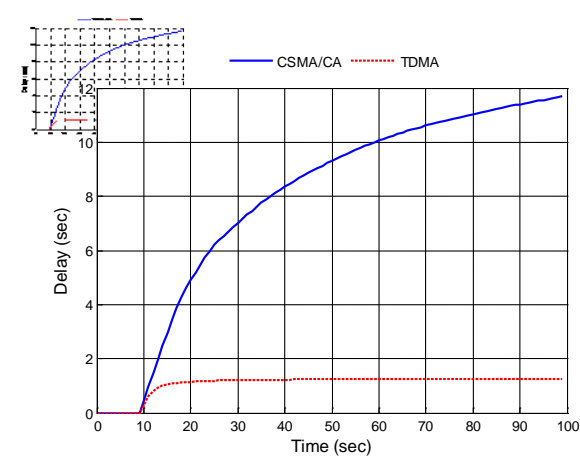

(b)

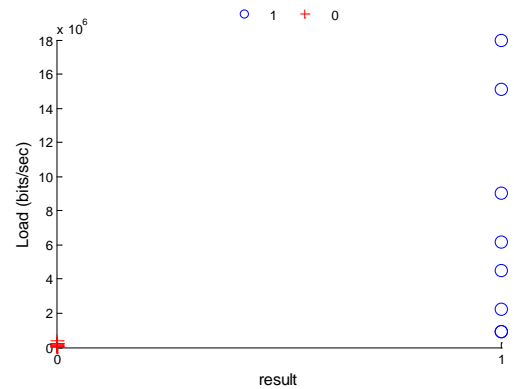

(d)

Fig. 11. Performance comparison between CSMA/CA and TDMA under 20 node scenarios. (a) throughput standard deviation. (b) delay. (c) CSMA/CA samples. (d) TDMA samples 


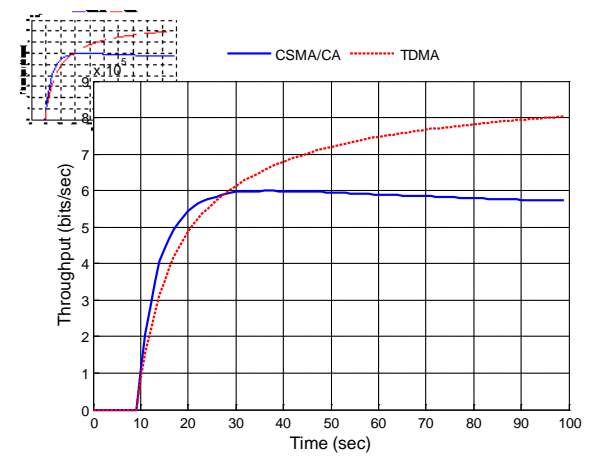

(a)

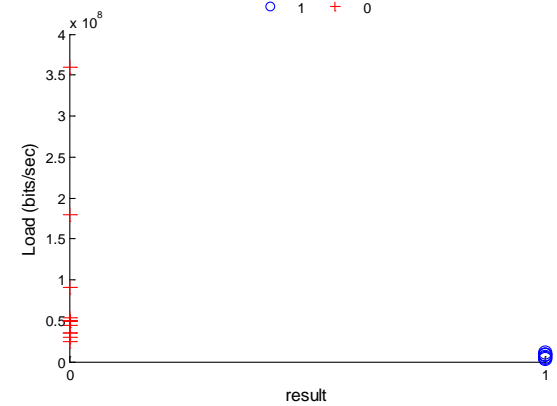

(c)

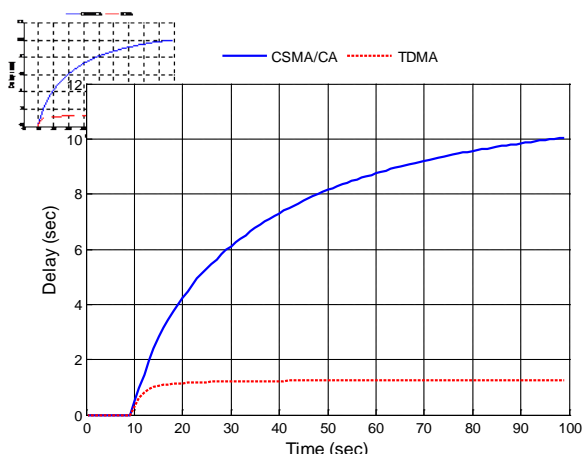

$0 \stackrel{(b)}{1}+0$

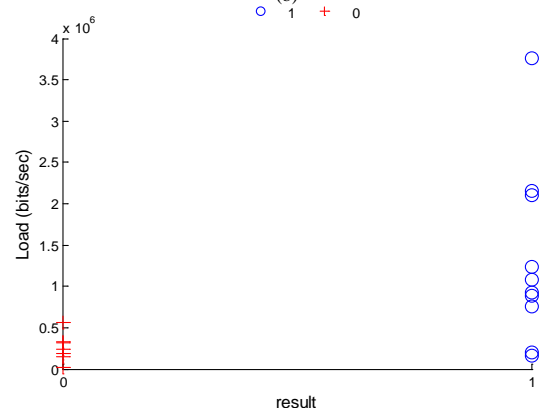

(d)

Fig. 12. Performance comparison between CSMA/CA and TDMA under 25 node scenarios. (a) throughput standard deviation. (b) delay. (c) CSMA/CA samples. (d) TDMA samples

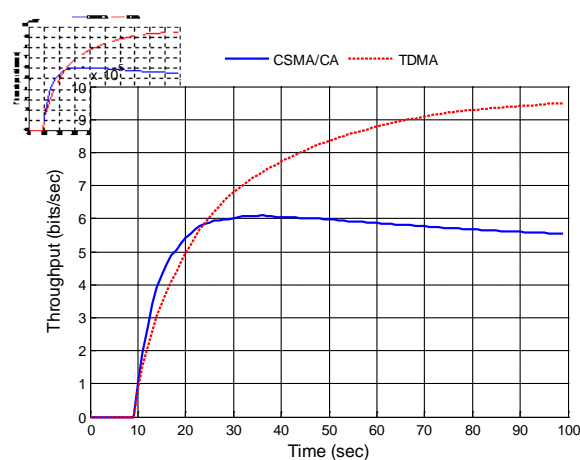

$\stackrel{(a)}{1}+0$

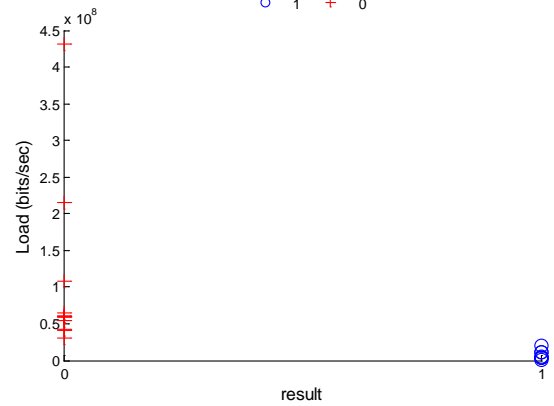

(c)

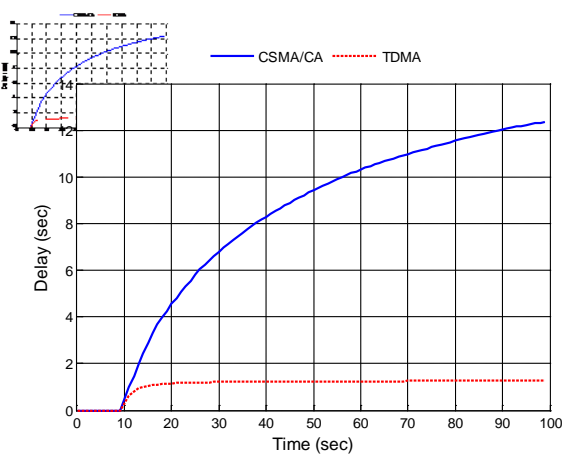

0 (b) $1+0$

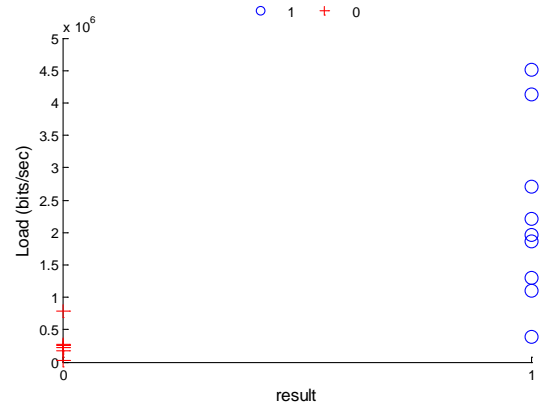

(d)

Fig. 13. Performance comparison between CSMA/CA and TDMA under 30 node scenarios. (a) throughput standard deviation. (b) delay. (c) CSMA/CA samples. (d) TDMA samples 


\subsection{Distribution Behavior under Variable Performance Curve}

In this part, we fix the number of the network node to 10 and the size of the data packet to 1000 bytes, and evaluate the load, i.e., the saturation curve of the throughput, of CSMA/CA and TDMA protocols in the network. To gain an explicit observation, we make a choice among the simulation result curves has been saturated, gradually and respectively. Sample distributions which around the saturation curves cross location of the two protocols are considered and shown in Fig. 14.

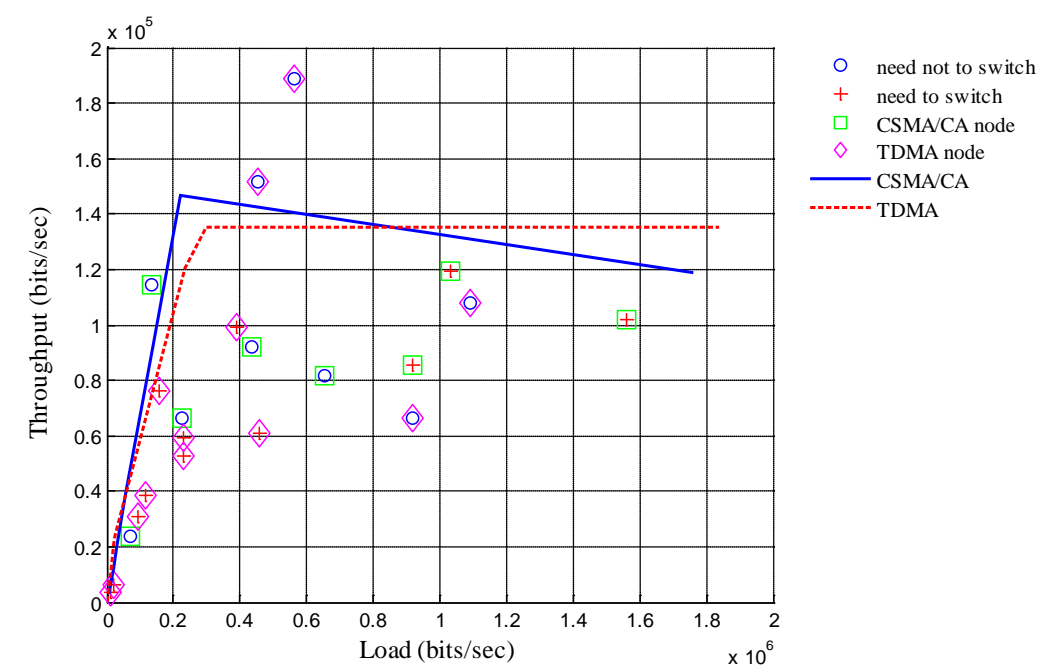

Fig. 14. Feature examples with Load-Throughput curve

With our extending protocol selection criterion in section 4.1, CSMA/CA protocol samples are classified to be switched when the performance gaps between CSMA/CA and TDMA increase with the load. This is because the nodes face more conflicts, and thus CSMA/CA protocol becomes less efficient. TDMA protocol samples are classified to be switched when the performance gaps between CSMA/CA and TDMA decrease with the load. This is because the performance curve of TDMA protocol has not reached the saturation region, which implies most of the time slots are not utilized by the nodes.

\section{Conclusion}

In this paper, we present a MAC protocol selection model in wireless sensor networks. By employing the SMO classification approach, the selection model can help sensor nodes to select MAC protocol that best fits the current network environment. We proposed a two-stage classification framework to complete the MAC protocol selection task. In our method, the competitive CSMA/CA protocol and the non-competitive TDMA protocol served as candidates. To rapidly provide a robust classification result, a feature data set for training and a test data set for evaluating were respectively established. Moreover, we investigated the contribution of different features to the classification result in the protocol selection model. Simulation results show that, the proposed classification method can achieve the better PoCC, and the MAC protocol selection model had the capability to select the appropriate MAC protocol that best fit the current network environment. In the future work, we plan to generalize the MAC protocol selection model, relying on which a classifier can be designed and applied to deal with the MAC protocol selection problem when multiple protocol 
candidates are included.

\section{References}

[1] N.C.Luong, D.T.Hoang, P.Wang, D.Niyato, D.I.Kim and Z.Han. "Data Collection and Wireless Communication in Internet of Things (IoT) Using Economic Analysis and Pricing Models: A Survey," IEEE Communications Surveys \& Tutorials, vol.18, no.4, pp. 2546-2590, 2016.

Article (CrossRef Link)

[2] A.De.Domenico, E.C.Strinati, and M-G.Di.Benedetto, "A Survey on MAC Strategies for Cognitive Radio Networks,” IEEE Communications Surveys \& Tutorials, vol.14, no.1, pp.21-44, 2015. Article (CrossRef Link)

[3] M. Jo, L. Han, N. Tan, and H. Peter "A Survey: Energy Exhausting Attacks in MAC Protocols in WBANs," Telecommunication Systems, vol.58, no.2, pp.153-164, 2015. Article (CrossRef Link)

[4] I.Rhee, A.Warrier, M.Aia, and J.Min, "Z-MAC: A hybrid MAC for wireless sensor networks," IEEE/ACM Transactions on Networking, vol.16, no.3, pp.511-524, 2008. Article (CrossRef Link)

[5] M.H.S.Gilani, I.Sarrafi, and M.Abbaspour, "An adaptive CSMA TDMA hybrid MAC for energy and throughput improvement of wireless sensor networks," Ad Hoc Networks, vol.11, no.4, pp.1297-1304, June.2013. Article (CrossRef Link)

[6] Z,Yang, Y.D.Yao, S.Chen, H.B.He, and D,Zheng, "MAC protocol classification in a cognitive radio network," in Proc. of 19th Annual Wireless and Optical Communications Conference, pp.1-5, 2010. Article (CrossRef Link)

[7] S.Q.Hu, Y.D.Yao, and Z,Yang, "MAC protocol identification using support vector machines for cognitive radio networks,” IEEE Wireless Communications, vol.21, no.1, pp.52-60, February.2014. Article (CrossRef Link)

[8] G.Naddafzadeh-Shirazi, P.-Y.Kong, and C.K.Tham, "Distributed Reinforcement Learning Frameworks for Cooperative Retransmission in Wireless Networks," IEEE Transactions on Vehicular Technology, vol.59, no.8, pp.4157-4162, 2010. Article (CrossRef Link)

[9] K.-H.Phung, B.Lemmensand, M.Goossens A.Nowe, L.Tran, and K.Steenhaut, "Schedule-based multi-channel communication in wireless sensor networks A complete design and performance evaluation," Ad Hoc Networks, vol.26, no.C, pp. 88-102, 2014. Article (CrossRef Link)

[10] H.Jang, SY.Yun, J.Shin, and Y.Yi, "Distributed Learning for Utility Maximization over CSMA-based Wireless Multihop Networks," in Proc. of the IEEE INFOCOM, 2014, pp.280-288. Article (CrossRef Link)

[11] G.Stamatakis, E.Z. Tragos, and A.Traganitis, "A Two-Stage Spectrum Assignment Scheme for Power and QoS Constrained Cognitive CSMA/CA Networks," in Proc. of the IEEE Globecom Workshops, 2015, pp.1-6. Article (CrossRef Link)

[12] S.Pandit, and G.Singh, "Backoff Algorithm in Cognitive Radio MAC Protocol for Throughput Enhancement," IEEE Transactions on Vehicular Technology, vol.64, no.5, pp. 1991-2000, 2015. Article (CrossRef Link)

[13] Y.Liu, V.O.K.Li, and K-C.Leung and L.Zhang, "Distributed multi-channel topology-transparent broadcast scheduling in ad hoc networks," in Proc. of the 2014 IEEE Wireless Communications and Networking Conference, 2014, pp. 1556-1561. Article (CrossRef Link) 
[14] M.Sami, N.K.Noordin, and M.Khabazian, "A TDMA-Based Cooperative MAC Protocol for Cognitive Networks With Opportunistic Energy Harvesting," IEEE Communications Letters, vol.20, no.4, pp. 808-811, 2016. Article (CrossRef Link)

[15] S.Zhou, Y.Song, Z.Wang, and Z.Wang, “Queue-MAC: A queue-length aware hybrid CSMA/TDMA MAC protocol for providing dynamic adaptation to traffic and duty-cycle variation in wireless sensor networks," in Proc. of the IEEE International Workshop on Factory Communication Systems, 2012, pp.105-114. Article (CrossRef Link)

[16] L.Sitanayah, Cj.Sreenan, and KN.Brown "A hybrid MAC protocol for emergency response wireless sensor networks,” Ad Hoc Networks, vol.303, no.20. pp.77-95, 2014.

Article (CrossRef Link)

[17] Q.Ye, W.Zhuang, L.Li, and P.Vigneron, "Traffic Load Adaptive Medium Access Control for Fully-Connected Mobile Ad Hoc Networks,” IEEE Transactions on Vehicular Technology, vol.20, no.4, pp.808-811, 2016. Article (CrossRef Link)

[18] M.Mihaylov, Y.A.Le Borgne, K.Tuyls, and A. Nowé, "Decentralised reinforcement learning for energy-efficient scheduling in wireless sensor networks," International Journal of Communication Networks \& Distributed Systems, vol.9, no.3/4, pp. 207-224, 2012. Article (CrossRef Link)

[19] K.M.Thilina, E.Hossain, and D.I.Kim I.Kakalou, "DCCC-MAC: A Dynamic Common Control Channel-Based MAC Protocol for Cellular Cognitive Radio Networks,” IEEE Transactions on Vehicular Technology, vol.65, no.5, pp.3597-3613, 2016. Article (CrossRef Link)

[20] G.Bianchi, L Fratta and M Oliveti, "Performance evaluation and enhancement of the CSMA/CA MAC protocol for 802.11 wireless LANs,” in Proc. of the IEEE PIMRC, Taipei, 1996, pp.392-396. Article (CrossRef Link)

[21] H.Wu, S.Peng and Y.Peng, "IEEE 802.11 distributed coordination function(DCF): analysis and enhancement," in Proc. of the IEEE ICC, New York, 2002, pp.605-609. Article (CrossRef Link)

[22] Y.Yi, G.de Veciana, and S.Shakkottai, "MAC Scheduling With Low Overheads by Learning Neighborhood Contention Patterns,” IEEE/ACM Transactions on Networking, vol.18, no.5, pp.1637-1650, 2010. Article (CrossRef Link)

[23] Y.Wei, H.John, and E.Deborah, "Medium access control with coordinated adaptive sleeping for wireless sensor networks,” IEEE/ACM Transactions on Networking, vol.12, no.3, pp.493-506, June 2004. Article (CrossRef Link)

[24] H.A.B.Salameh, M.M.Krunz, and O.Younis, "MAC Protocol for Opportunistic Cognitive Radio Networks with Soft Guarantees," IEEE Transactions on Mobile Computing., vol.8, no.10, pp.1339-1352, October 2009. Article (CrossRef Link)

[25] C.X.Zhu, and M.S.Corson, "A Five-Phase Reservation Protocol for mobile Ad Hoc Networks," Wireless Networks, vol.7, no.4, pp.371-384, 2001. Article (CrossRef Link)

[26] T.Chen, H.Zhang, G.M.Maggio, and I.Chlamtac, "Cogmesh: A cluster-based cognitive radio network,” in Proc. of the Symposium on Dynamic Spectrum Access Networks, Dublin, Ireland, November 2007, pp. 168-178. Article (CrossRef Link)

[27] G.Lu, B.Krishnamachad, and CS.Raghavendra, "Adaptive energy-efficient and low-latency MAC for tree-based data gathering in sensor networks," Wireless Communications \& Mobile Computing, vol.7, no.7, pp.863-875, 2007. Article (CrossRef Link)

[28] A.Zadeh, "Fuzzy sets”, Information and Control, vol.8, no.1, pp.338-353, 1965.

Article (CrossRef Link) 
[29] G.Y.Wang, and Q.H.Zhang, “Granular computing based cognitive computing,” in Proc. of the 8th IEEE International Conference on Cognitive Information Conference, pp.155-161, 2009.

$\underline{\text { Article (CrossRef Link) }}$

[30] Ross Quinlan, C4.5:Programs for Machine Learning, Morgan Kaufmann Publishers, San Mateo, CA, 1993. Article (CrossRef Link)

[31] George H.John, and Pat Langley, “Estimating Continuous Distributions in Bayesian Classifiers,” in Proc. of the 11th Uncertainty in Artificial Intelligence Conference, pp.338-345, 1995.

Article (CrossRef Link)

[32] JC.Platt, "Sequential Minimal Optimization A Fast Algorithm for Training Support Vector Mechines,” Advances in Kernel Methods-support Vector Learning, vol.208, no.1, pp.212-223, 1998. Article (CrossRef Link)

[33] Leo Breiman, “Random Forests,” Machine Learning, vol.45, no.1, pp.5-32, 2001.

Article (CrossRef Link) 

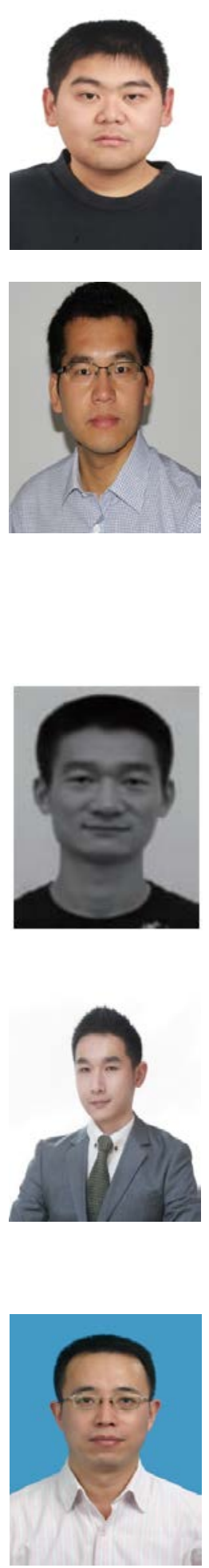

Mu Qiao received his B.S. degree in Information Engineering from the Hunan Agricultural University, Changsha, China in 2011. And he obtained the M.S. degree in Software Engineering from the National University of Defense Technology (NUDT), Changsha, China in 2013. He is currently a Ph.D. candidate with the Department of Communication Engineering, College of Electronic Science, NUDT. His research interests include MAC protocol, Machine Learning, Cognitive Radio Networks.

Haitao Zhao received his M.S. and Ph.D. degree in information and communication engineering both from National University of Defense Technology (NUDT), Changsha, China in 2004 and 2009, respectively. He is currently an Associate Professor in College of Electronic Science, NUDT. Prior to this, from April 2008 to July 2009 he worked as a visiting research associate in the Institute of ECIT, Queen's University of Belfast, UK. And from 2014 to 2015, he conducted postdoctoral research at Hong Kong Baptist University. He is now a member of IEEE, ACM, a mentor member for IEEE 1900.1 standard group, and a member of World-wide University Network (WUN) Cognitive Communications Consortium. And he has served as a TPC members of IEEE ICC'14-17, Globecom'16, APCC'16 and guest-edited several international journal special issues on cognitive radio networks. His main research interests include cognitive radio networks, self-organized networks and cooperative communications.

Shengchun Huang received the B.S. and M.S. degrees from NUDT, China in 2005 and 2008, respectively. From Oct. 2009 to Oct. 2011, he was a visiting Ph.D. student at the University of British Columbia, Canada. His research interests include broadband wireless access network, radio resource management. Email: huangsc@nudt.edu.cn

Li Zhou received his B.S., M.S. and Ph.D. degrees from National University of Defense Technology (NUDT), China in 2009, 2011 and 2015 respectively. From Sept. 2013 to Sept. 2014 he worked as a visiting scholar at The University of British Columbia, Canada. He is currently an assistant professor at College of Electronic science, NUDT, China. His research interests are in the area of software defined radios (SDRs), software defined networks (SDNs) and heterogeneous networks (HetNets). Dr. Zhou served as a TPC member in IEEE CIT 2017, keynote speaker in ICWCNT 2016 and co-chair in ITA 2016. His research contributions have been published and presented in more than 20 prestigious journals and conferences, such as IEEE Transactions on Vehicular Technology, Ad Hoc Networks, WInnComm 2017, IEEE INFOCOM 2015 and IEEE GLOBECOM 2014.

Shan Wang associate professor with College of Electronic Science, National University of Defense Technology (NUDT). He received his B.S. and Ph.D. degrees from NUDT, China in 2000 and 2006, respectively. He worked as a postdoctor in University of Montreal, Canada from Sept. 2010 to Sept. 2011. His research interests include protocol analysis, modeling and design, ad hoc networks, networks simulation and optimization. 